\title{
Micropattern properties and presentation conditions influencing visual texture discrimination
}

\author{
RICK GURNSEY and ROGER A. BROWSE \\ Queen's University, Kingston, Ontario, Canada
}

\begin{abstract}
Texton theory defines textons as such local features as elongated blobs, terminators, and line crossings. The theory states that effortless visual texture discrimination may occur between two regions only if they differ in texton density, irrespective of the spatial relationships among textons. It is argued here that line-crossing and terminator textons have not been demonstrated to function independently of configurational differences between micropatterns. Four experiments tested whether line crossings and terminators actually elicit effortless discrimination independently of configurational differences. Subjects were required to detect a disparate textured region embedded in an unpredictable quandrant of a textural display. The textural displays were presented for brief durations, ranging from 67 to $167 \mathrm{msec}$, and followed by a random dot mask. In general, when configuration was controlled, micropatterns differing in terminators and line crossings elicited relatively poor discrimination. Ease of discrimination, as measured by the probability of a correct detection, was largely associated with differences in micropattern size (measured by the minimum enclosing circle). In addition, for certain texture pairs, ease of discrimination depended crucially upon which member of the pair formed the embedded region and which formed the background. This foreground/background asymmetry was also related to size differences between micropatterns forming the textures. In many cases, performance improved at longer stimulus durations, although the rate of increase and absolute level of performance depended on the particular texture pair being tested. Qualitative differences in performance between naive subjects and a highly practiced subject were also observed.
\end{abstract}

Texture discrimination and segmentation have generated a great deal of interest in recent years in both the psychological literature (e.g., Beck, 1982; Beck, Pradzny, \& Rosenfeld, 1983; Caelli, 1982, 1985; Caelli \& Moraglia, 1985; Enns, 1986; Grossberg \& Mingolla, 1985; Julesz, 1981, 1984; Julesz \& Bergen, 1983; Nothdurft, 1985a, 1985b, 1985c) and the machine vision literature (e.g., Haralick, 1979; Laws, 1980; Pentland, 1984). The research described in this paper was intended primarily as an evaluation of Julesz's texton theory, but the empirical results have implications for theories of texture segmentation in general.

Julesz (1984) has proposed a theory of human vision that takes the form of three heuristics. The first heuristic adheres to the distinction between attentive and preattentive vision popularized by Neisser (1967). According to texton theory, the preattentive visual system distributes processing over a broad portion of the visual field but is

This research was supported by a grant from the Natural Sciences and Engineering Research Council of Canada (A2427). We would like to thank Peter Dodwell, Doug Mewhort, David Fleet, and Terry Caelli for their comments on various drafts of this paper. We would also like to thank Mike Jenkins for the use of his Symbolics LM3600 computer. Correspondence may be addressed to either Rick Gurnsey, Department of Psychology, or Roger Browse, Department of Computing and Information Science, Queen's University, Kingston, Ontario, Canada K7L 3N6. sensitive only to rather simple image characteristics, such as line orientation. The attentive visual system operates within a restricted spatial aperture but is capable of establishing relationships between simple image features, such as whether two line segments are arranged so as to form an L or a T (Bergen \& Julesz, 1983). As with feature integration theory (Treisman \& Gelade, 1980), texton theory holds that the spatial locus of attentive processing can be repositioned several times wtihin an eye fixation. The second heuristic asserts the existence of local image features, called textons, that are detected by the preattentive visual system. The three classes of textons associated with texture discrimination are: (1) elongated blobs with particular intensities, orientations, aspect ratios, and scale, (2) terminators (free ends of lines), and (3) line crossings. The third heuristic states that "preattentive vision directs attentive vision to the locations where differences in textons or in the densities (numbers) of textons occur, but ignores the positional relationships between textons" (Julesz, 1984, p. 590).

Texton theory does not provide formal definitions of textons themselves. Instead, the characteristics of textons must be inferred from examples given in expositions of the theory. Consider, for example, the L-, T-, and +shaped micropatterns that form the textures shown in panels $a$ and $b$ of Figure 1. Each of these micropatterns is composed of the same line segments. According to the 


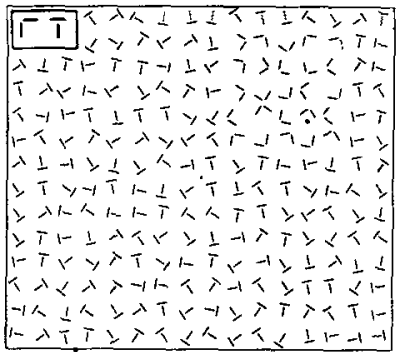

(a)

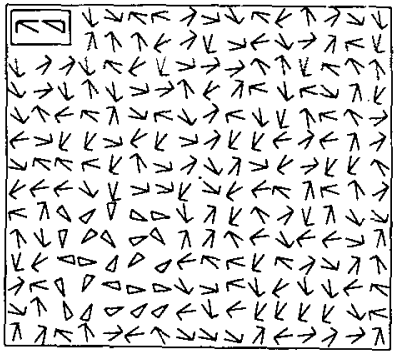

(d)

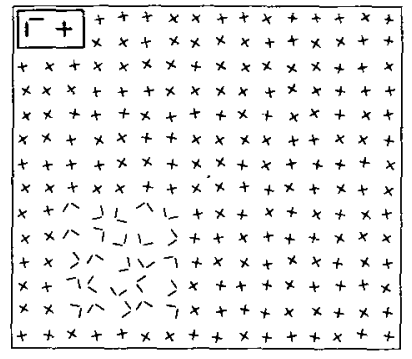

(b)

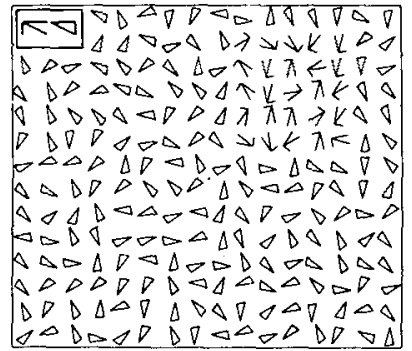

(e)

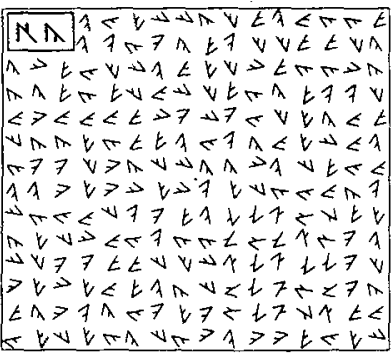

(c)

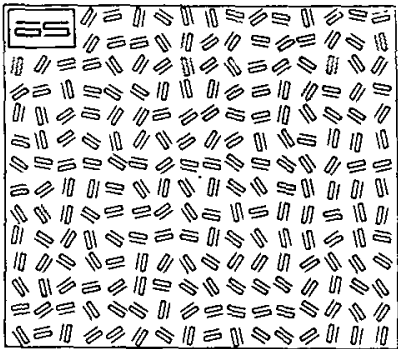

(f)

\begin{abstract}
Figure 1. Examples of discriminable and indiscriminable micropattern pairs. Displays similar to those shown in Panels b, d, and e have been reparted by Julese (1984) to be strongly discriminable. The remaining displays have been reported $\mathbf{s}$ being indiscriminable.
\end{abstract}

theory, the same set of line-segment and terminator textons would be activated in all textured regions, whether composed of Ls, Ts, or $+s$. The theory predicts that the textures shown in Figure 1a will be poorly discriminated because they differ only in terms of the spatial relationships between line-segment and terminator textons. On the other hand, the L- and +-shaped micropatterns shown in Figure 1b, should be quite easy to discriminate, according to the theory, because of the unshared linecrossing texton (Julesz \& Bergen, 1983).

Central to texton theory is the claim that textures are analyzed into elementary features (textons) and that differences in texton density or identity between neighboring regions give rise to discrimination. An alternative proposal, due to Marr (1982) and Beck (1982; Beck et al., 1983), is that a stage of synthesis follows the initial decomposition of the image into elementary features. On such a view, simple features, such as oriented blobs, may be linked (unitized) according to such Gestalt principles as similarity, proximity, or good continuation, to form higher order units that have such properties as scale and orientation associated with them. Beck and Marr suggest that textural segmentation may depend on the properties of these higher order units. Different arrangements (configurations) of the same line segments may result in the construction of molar units, what Marr (1982) called "place tokens," that have different descriptions. For example, the radius of the minimum enclosing circle of the $\mathrm{L}$ is approximately 1.41 times greater than that of the + but only 1.13 times greater than that of the $T$. On the basis of this particular description of micropattern size, $\mathrm{L}$ could be more discriminable from + than $\mathrm{T}$. Texton theory must claim that the discriminability of the $\mathrm{L}$ - and +-shaped micropatterns is independent of configurational differences (if the claim that line crossings control discrimination is to be maintained). However, this independence has not been demonstrated. ${ }^{1}$

It is not necessary to assume that line segments are independently detected and then linked for one to view different arrangements of the same line segments as contributing to discrimination. Differences in line-segment arrangement would be expected to elicit different responses from simple filters. For example, because $\mathrm{L}$ and + are enclosed by different minimum circles, they would differently stimulate simple center-surround operators (e.g., Marr \& Hildreth, 1980) or "simple-orientation selective units in the visual cortex" (Bergen \& Julesz, 1983 , p. 861). Furthermore, Caelli (1985) has demonstrated computationally that L- and +-shaped micropatterns may be segmented on the basis of the responses of simple orientation selective filters-that is, an existence proof that line-crossing detectors are not necessary for discrimination. There are, then, alternative accounts of the ease with which Figures $1 \mathrm{a}$ and $1 \mathrm{~b}$ can be discriminated that do not require the existence of line-crossing detectors. We will argue below that texton theory itself does not require the line-crossing texton or even the terminator texton.

The claim that line crossings form the basis of discrimination in Figure $1 \mathrm{~b}$ implies that $\mathrm{L}$ and + do not stimulate different blob-texton detectors in spite of having different global descriptions in terms of size and shape. ${ }^{2}$ 
However, the theory is inconsistent on this point. The micropatterns shown in Figure 1c are reported to be preattentively indiscriminable (Julesz, 1981). According to texton theory, poor discriminability in this case is due to the identical line-segment and terminator composition of the micropatterns. With practice, however, textures composed of these two patterns do become discriminable (Julesz, 1981,1984 ). Julesz (1984) offers the following comment on this point:

My explanation is that the closed and open dual elements stimulate different elongated detectors and thus are different textons. With long practice one learns to pay attention to these weakly stimulated texton detectors tuned to different sizes. (p. 604)

If different spatial arrangements of line segments elicit different responses from blob-texton detectors, there is no need to hypothesize the existence of line-crossing textons to explain the discriminability of textures composed of Ls and $+s$. The logic by which terminators were inferred as textons is similarly undermined because all available examples of discriminable textures composed of terminator-differing micropattern pairs have concomitant configurational differences (e.g., panels $d$ and $e$ of Figure 1). Texton theory could explain discriminability of such textures in terms of the responses of large-scale blob-texton detectors. Conversely, the micropattern pair shown in panel $f$ of Figure 1 is often used to argue that micropatterns that agree in terminator and line-segment number are indiscriminable. Based on the apparent tuning characteristics of blob-texton detectors, failure of discrimination could equally be attributed to the overall similarity of the two configurations.

Texton theory offers a second way in which different arrangements of line segments result in the stimulation of different blob-texton detectors. Most micropatterns used by Julesz to illustrate texton theory are composed of black line segments on a white background, and textons have been defined in terms of these line segments. However, Julesz (1984) points out that micropattern pairs such as those shown in panels $d$ and e of Figure 1 differentially "stimulate white elongated blob textons" (p. 593). Texton theory, then, could attribute discriminability to differences in large white blobs rather than terminators. Given this uncertainty about the role played by terminators and line crossings in texture discrimination, we performed experiments to compare the discriminability of micropattern pairs that differed in configuration with those that minimized configurational differences while differing in line-crossing and/or terminator composition. ${ }^{3}$

As mentioned earlier, texton theory distinguishes between preattentive and attentive visual processes. Texton theory states that the spatial locus of attentive processing can be repositioned at about 50-msec intervals. Converging evidence that attention may be repositioned within a fixation comes from the work of Posner and others (Posner, 1980; Shulman, Remington, \& McLean, 1979; Tsal, 1983). Ullman and Jolicoeur (Jolicoeur, 1986; Jolicoeur,
Ullman, \& Mackay, 1986; Ullman, 1984) also argue for the existence of fast serial processes capable of establishing spatial relationships between elements of a base representation.

The availability of a quickly repositionable "form processor" must be taken into account in texture discrimination experiments when textures are composed of micropatterns that are clearly distinguishable when attention is focused. If the location of the disparate region was predictable or exposure duration was sufficiently long, then two textures (composed of micropatterns) could be discriminated by quickly repositioning the locus of attentive processing. In these cases, it could not be determined if discrimination was based on properties of the textures as wholes or on the examination of individual micropatterns. Therefore, following Beck and Ambler (1973; see also Enns, 1986; Fox \& Mayhew, 1979; Julesz, 1980; Olson \& Attneave, 1970), subjects in the experiments described below were required to discriminate two textures arranged such that the disparate texture was embedded within one of the four quadrants of the display. The location of the disparate region was unpredictable from trial to trial and a range of exposure durations was covered. ${ }^{4}$ Such a paradigm should reduce the effectiveness of a search strategy.

As an alternative to the strict attentive/preattentive dichotomy, Bergen and Julesz (1983) suggest that the extent of the image that can be processed in parallel is related to the magnitude of the differences between regions. (Bergen and Julesz's suggestions are very similar to suggestions made by Beck and Ambler, 1972, p. 38.) If largescale differences exist between regions, then these are detected with a wide processing aperture. If only small-scale differences exist, then the aperture must be narrowed and, in the limiting case, individual micropatterns must be scrutinized. Since narrowing the aperture and shifting processing location are time-consuming processes, the average inspection time required to reach some criterion level of performance should, by their model, reflect the scale of the differences between regions.

It would seem obvious that a region of texture $A$ embedded within a background of texture $B$ should elicit the same discriminability as a region of texture $B$ embedded within a background of texture A. This intuition is explicit in Beck's (1982, p. 307) discussion of textural segmentation: "Textural regions are discriminated from each other and it is not meaningful to say region $A$ is more readily discriminated from region $B$ than is region $B$ from region A." However, there are circumstances in which asymmetries may be expected to arise: "One may ... expect textural segmentation in which disparate figures are embedded in background figures to exhibit certain asymmetries."' Beck's examples (Beck, 1982, Figure 15.11, and Beck, 1973, Figures 5i and 5j) show two types of micropatterns dispersed through the same region, for example, short vertical lines dispersed through a region of long vertical lines. Beck is arguing that two textural regions do not give rise to an asymmetry, whereas embed- 
ded figures do produce asymmetries. The question we are asking involves embedded regions and so, from this account, it is not clear whether or not embedded regions should give rise to an asymmetry.

Examples of asymmetries in a visual search task have been reported by Treisman and Souther (1985) and Julesz (1981). Treisman and Souther found that the time required to detect a circle with an intersecting line embedded in a background of circles was independent of the number of distracting circles, whereas the time required to detect a circle in a background of circles with intersecting lines did depend on the number of background distractors. Julesz noted that, in a detection task, the patterns shown in the insets of Figures 1c and 1d (arrow and triangle) elicited asymmetrical detection. Detecting three arrows in a background of 32 triangles was equally easy regardless of whether the three arrows were placed in the same neighborhood or dispersed throughout the distractors. Detecting three triangles in a background of 32 arrows, however, was easier if the triangles were presented in the same neighborhood. Julesz's explanation was that in a field of terminators, a small area with no terminators had some probability of occurring by chance. Therefore, the area of low terminator density had to be rather large before it was detected as a nonchance event. The general claim seems to be that if there is an asymmetry in the texture discrimination task, it should be the micropattern with more "textons" that has the greater probability of being discriminated. Therefore, in all experiments reported here we investigated the possibility that asymmetries exist in a task requiring the discrimination of two spatially disjoint textures.

\section{EXPERIMENT 1}

In the first experiment, we investigated the discriminability of textures composed of Ls, Ts, and $+s$ and three additional patterns derived from these. The six micropattern pairs used were those shown in Figure 2. Note that the $\mathrm{L}$ - and T-shaped micropatterns shown in Figure 2 have no gaps between their component lines as do the Ls and Ts in Figure 1. Henceforth, when we refer to Ls and Ts, we will mean the Ls and Ts shown in Figure 2.

Because obvious mnemonics do not exist for many of the micropatterns used in the following experiments, we adopt the convention illustrated in Figure 2 to refer to the different pairs. The pair consisting of $\mathrm{L}$ - and +-shaped micropatterns will be referred to as Pair $1.1, \mathrm{~L}-$ and $\mathrm{T}$ shaped micropatterns as Pair 1.2, and so on. We shall refer to the case of $L s$ embedded in $+s$ as Condition 1.1.1, $+\mathrm{s}$ embedded with Ls as 1.1.2, and so on. Wherever possible, however, obvious mnemonics will be used.

The first three micropattern pairs (involving $\mathrm{L}-,+$,and T-shaped patterns) have been investigated extensively by Beck (1966, 1967; Beck \& Ambler, 1972, 1973) and more recently by Bergen and Julesz (1983) in the context of a search task. Bergen and Julesz found that a single $\mathrm{L}$ embedded in a background of $35+\mathrm{s}$ could be detected

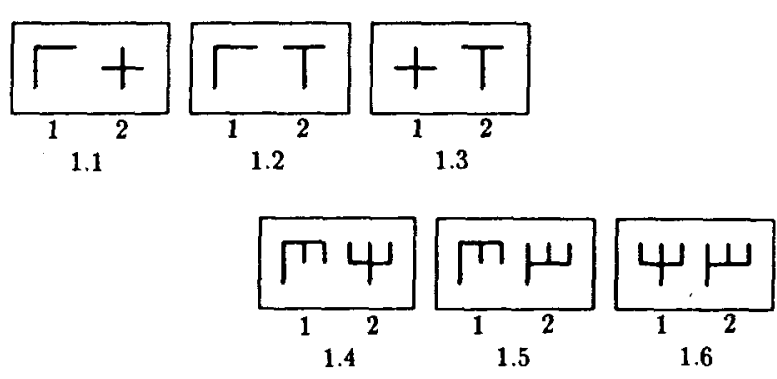

Figure 2. The six micropattern pairs used in Experiment 1.

with almost perfect accuracy at a stimulus onset asynchrony (between onset of stimulus and mask) of $150 \mathrm{msec}$, whereas picking an $\mathrm{L}$ out of a background of $35 \mathrm{Ts}$ elicited only chance performance at the same stimulus onset asynchrony. The texton theory interpretation of this result is that there is a large texton difference between $\mathrm{L}$ and + and an insignificant difference between $\mathrm{L}$ and $\mathrm{T}$. The $\mathrm{T}-+$ combination was not considered.

The investigation of Pairs 1.1 and 1.2, then, should establish certain baselines against which the discriminability of other micropattern pairs may be compared. Bergen and Julesz found Pair 1.1 (L-+) to be highly discriminable in the search task, and so it would be expected to elicit very good discrimination in the texture discrimination task as well. Pair 1.2 (L-T), on the other hand, elicited poor discriminability in the search task. Julesz (1984) argued that attentive vision was required to detect an L embedded within 35 Ts. Therefore, the pattern of results obtained for the $\mathrm{L}$ - and $\mathrm{T}$-shaped micropatterns should be characteristic of preattentively indiscriminable micropatterns. Pair $1.3(+-\mathrm{T})$ presents an interesting case. The existence of the unshared linecrossing texton suggests that Pair 1.3 should be as easily discriminated as Pair $1.1(\mathrm{~L}-+)$ if the salience of a texton is independent of its context. However, there is only one unshared terminator in the case of Pair 1.3, whereas there are two in Pair 1.1. If discriminability is a function of the total number of unshared textons, Pair 1.3 should be somewhat more difficult to discriminate than Pair 1.1. In this case, the salience of a texton is context dependent, as suggested by Enns (1986; see also Beck, 1982).

The remaining three micropattern pairs were devised to test the notion that line crossings were important in texture discrimination. Pairs $1.4,1.5$, and 1.6 may be seen as having been derived from $\mathrm{L},+$, and $\mathrm{T}$ by the addition of two short lines to each. Alternatively, these three patterns may be seen as all having the same basic " $E$ " configuration with one short line added. Because Pairs 1.4, 1.5 , and 1.6 share the basic " $E$ " structure, we expected to minimize the extent to which discriminability could be attributed to configurational differences. If line crossings were of fundamental importance, Pairs 1.4 and 1.6 should be more discriminable than Pair 1.2, which does not differ in terms of line crossings. If line crossings were not of fundamental importance, we would expect Pairs 1.4 and 
1.6 to be poorly discriminated because of overall configurational similarity.

\section{Method}

Subjects. Twelve subjects were selected at random from among graduate and undergraduate students in psychology and computer science at Queen's University at Kingston who had volunteered to take part in this series of experiments. Each subject was paid $\$ 8$ for participation. All subjects reported normal or corrected-tonormal vision.

Materials and Apparatus. The texture displays were constructed by the placement of 14 (vertical) $\times 16$ (horizontal) micropatterns at random orientations on a square grid. A slight positional jitter was added to each micropattern. The micropatterns appeared as black characters on a white background. The disparate region was a five $\times$ five square placed in one of the four quadrants. Except for the borders and insets, the structure of the displays shown in Figure 1 is identical to those used in the experiment.

The displays were presented on a color television monitor that was under the control of a Symbolics 3600 Lisp Machine. The subjects viewed the display binocularly at a distance of $1.5 \mathrm{~m}$ from the screen. At this distance, the display subtended $11^{\circ}$ vertical $x$ $12.6^{\circ}$ horizontal.

Procedure. At the beginning of each trial, a small dot appeared in the center of the screen. The subject fixated the dot and, when ready, pressed a button to initiate the trial. The trial consisted of the presentation of the textured field, which remained on for 67 , 100,133 , or $167 \mathrm{msec}$, followed immediately (i.e., an interstimulus interval of $0 \mathrm{msec}$ ) by a dense field of random dots, which remained on for $500 \mathrm{msec}$. The subjects' task was to detect the disparate region. Immediately after the offset of the masking dots, a box divided into four quadrants appeared on the screen. The subjects indicated their decisions by moving a mouse into the quadrant of the box corresponding to the location at which the disparate region was judged to have been, then pushed a button. The subjects were instructed to guess when they were uncertain of the location of the disparate region.

Design. There were six micropattern pairs, and the members of each pair served equally often as the disparate region. All combinations were tested equally often under all stimulus durations. A control condition was included in which the foreground and background regions involved the same micropattern that was chosen at random from those used in the actual test trials.

The experiment may be conceptualized--although not analyzedas a $7 \times 2 \times 4$ factorial design with seven micropattern pairs, including the control pair, two foreground/background conditions to determine which element of the pair served as the disparate region, and four stimulus durations. Each subject was presented with the resulting 56 stimulus conditions twice in each quadrant for a total of 448 trials. The order of presentation was divided into eight blocks. Each of the 56 cells was presented once in each block in a random order. The quadrant in which a particular configuration of conditions appeared was chosen at random subject to the above constraint that each stimulus condition appear in each quadrant twice during the course of the experiment. For control conditions, one of the quadrants was designated as the correct quadrant in the same manner as on all other trials.

Practice trials. Each subject was given 56 practice trials to become familiar with the procedure. The practice trials were broken down into four blocks of 14 trials each. During each practice block, the 14 different micropattern by foreground/background conditions were presented in random order. The disparate quadrant was chosen at random. In the first practice block, the textured display remained on until the subject had located the disparate quadrant. When the disparate quadrant had been located, the subject pressed a button to erase the display and responded as described above. An arbitrary choice was required for the control condition. The remaining three blocks of practice trials were presented exactly like experimental trials except that the stimulus durations were $500 \mathrm{msec}$ on Block 2, $233 \mathrm{msec}$ on Block 3, and $100 \mathrm{msec}$ on Block 4. Thus, the stimulus durations were gradually brought down into the range used during the experiment. The entire experiment, including practice trials, took between $45 \mathrm{~min}$ and $1 \mathrm{~h}$ to complete.

\section{Results and Discussion}

Analysis. For each of the 56 stimulus conditions in the experiment, the number of correct responses was used as the dependent measure. The analysis was carried out using a set of orthogonal weights to test the main effect of micropattern pairs, the foreground/background effect for each of the micropattern pairs, and the linear, quadratic, and cubic trends in the stimulus-duration effect for each micropattern $\times$ foreground/background condition.

Micropattern pairs. The main effect of micropattern pair was significant $[F(6,66)=68.98, p<.0001]$. The overall discriminability of each micropattern pair, excluding the control condition, is summarized in Figure 3. Planned comparisons that were not part of the orthogonal set revealed that Pairs 1.1 through 1.4 were significantly different from the control condition $[F(1,11)=178.6$, $31.53,90.83,29.14$ for Pairs 1.1 through 1.4, respectively; all $p \mathrm{~s}<.001$; Pair 1.6 was also different from the control condition $[F(1,11)=9.53, p<.02]$.

The observed pattern of results for Pairs 1.1 through 1.3 is consistent with what might be predicted from texton theory. Pair $1.1(\mathrm{~L}-+)$, which differed by two terminators and one line crossing, was the most discriminable; Pair 1.2 (L-T), which had only one unshared terminator, was the most difficult of the three. Pair 1.3

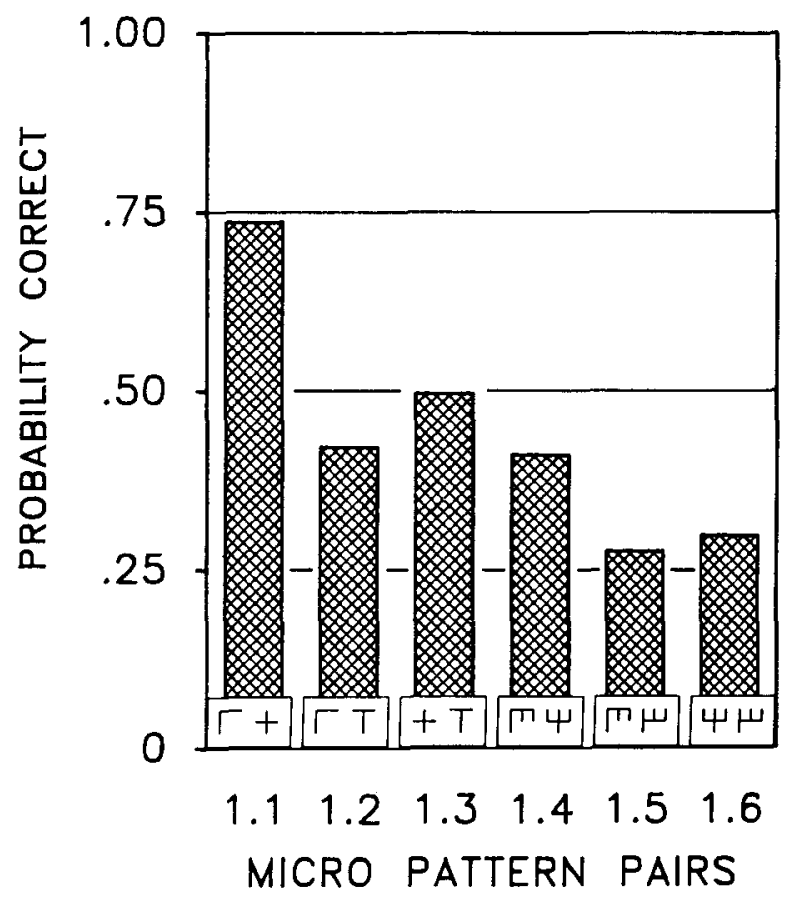

Figure 3. The overall discriminability of the micropattern pairs used in Experiment 1, collapsed over stimulus duration and the foreground/background factor. 
(+-T), which differed by one terminator and one line crossing, elicited intermediate discriminability, which is consistent with Enns's (1986) suggestion that the salience of a texton is context dependent. The mean overall probabilities of discrimination for Pairs 1.1 to 1.3 were .736, .421 , and .496 , respectively. Contrary to texton theory, however, Pair 1.4 was not numerically different from Pair 1.2 (.41 vs. .421 , respectively) and Pair 1.6 was numerically worse than Pair 1.2 (.297 vs. .421 , respectively). Thus, when configurational differences between micropatterns are minimized, micropatterns with linecrossing differences are no more disciminable than the L-T pair considered by Julesz to be preattentively indiscriminable. In other words, if Pair 1.2 is considered to be preattentively indiscriminable, then so must Pairs 1.4 and 1.6, which show equivalent or worse performance. However, it could be argued that the addition of the two short lines in Pairs 1.4 and 1.6 reduced the salience of the line-crossing texton. In this case, a "textons-in-context"' (Enns, 1986) version of texton theory would have to be adopted.

On the other hand, it is interesting to note that performance is related to differences in micropattern size (measured by the minimum enclosing circle). The ratios of the minimum enclosing circles of the larger to the smaller of the two patterns for Pairs 1.1 to 1.6 are 1.41, 1.13, $1.25,1.13,1.0$, and 1.13 , respectively. The overall discriminabilities of Pairs 1.1 to 1.6 were $.736, .421, .496$, $.41, .275$, and .297 , respectively. The correlation coefficient for these two sets of numbers is .96 . The implication is that something associated with differences in micropattern size (based on the minimum enclosing-circle descriptor) contributes to performance.

Foreground/background effect. The breakdown of the micropattern pairs into their foreground/background components is shown in Figure 4. For Pairs 1.1 through 1.4, there are striking asymmetries, indicating that the discriminability of a texture pair depends crucially on which of the two patterns is the foreground and which is the background. The planned contrasts showed that the effect was significant for Pairs 1.1 through $1.4[F(1,11)=$ $26.7,33.9,20.86,17.95$, respectively; all $p$ s $<.005$ ]. Pairs 1.5 and 1.6 and the control group did not show the effect, although Pair 1.6 approached significance $[F(1,11)$ $=4.41, p<.06]$. Again, it is interesting to note the role of size differences. When an asymmetry exists, it is when the larger of the two patterns forms the disparate region that discrimination is best. It is also significant to note that, contrary to suggestions made by Julesz (1984; Bergen \& Julesz, 1983; Julesz \& Bergen, 1983), +s embedded with Ls are actually less discriminable than Ls embedded with Ts (.536 vs. .576 , respectively). Although this result is inconsistent with our impressions from casual viewing, we will defer speculation about what causes the asymmetry. For the moment, we will say only that it cannot be just feature differences between adjacent regions that determine discrimination (cf. Julesz, 1984).

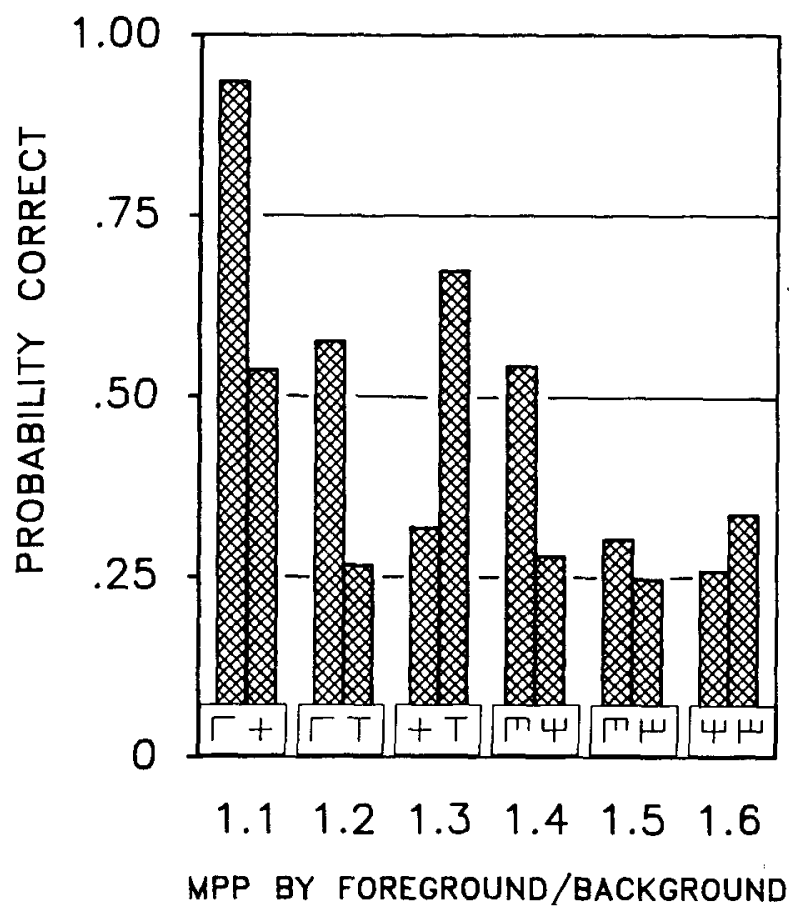

Figure 4. The discriminability of micropattern pairs used in Experiment 1 broken down in terms of which member of the pair played the role of the foreground.

As mentioned earlier, texton theory seemed to predict that if there was an asymmetry at all it should be the texture with the greater number of textons that is more discriminable. The reverse is true of these results. The micropattern with more terminators and/or line crossings was the more poorly discriminated member of the pairs that showed the asymmetry. Given the apparent robustness of the effect, it is remarkable that, in 25 years of experimentation, the asymmetry has not emerged as a fundamental issue to be explained by theories of texture discrimination.

Stimulus-duration-trend analysis. Results for the entire design (excluding control groups) are summarized in Figure 5 in terms of performance for each of the 12 micropattern pair $\times$ foreground/background conditions as a function of stimulus duration. Significant linear trends were found for Conditions 1.1.2, 1.2.1, 1.3.1, 1.3.2, and $1.4 .1[F(1,11)=15.19,5.24,10.94,16.51,11.40$, respectively; all $p s<.01]$. The linear trend for Condition 1.1.1 approached significance $[F(1,11)=4.66$, $p<.06]$, and there was a significant quadratic trend $[F(1,11)=9.14, p<.02]$, probably reflecting a ceiling effect.

We examined the change in performance as a function of stimulus duration because we wished to obtain some measure of the degree of the perceptual difference between two textured regions. The Bergen and Julesz (1983) variable-aperture model suggests that high accuracy at short stimulus durations implies large-scale perceptual 

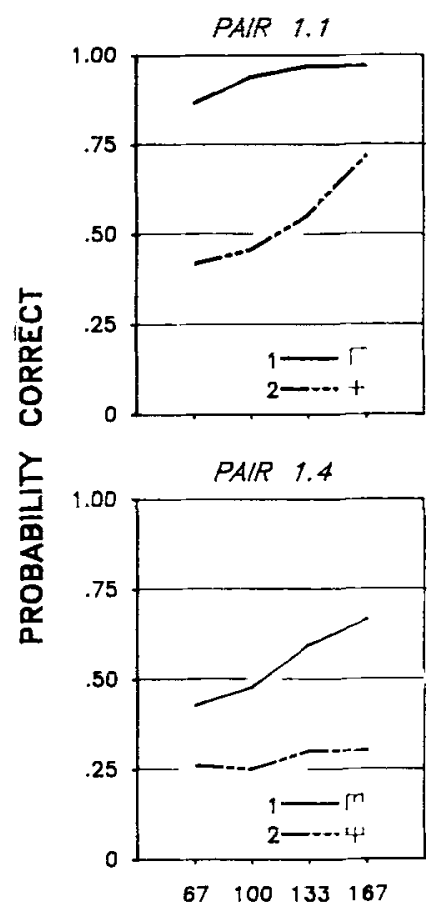

PAIR 1.2

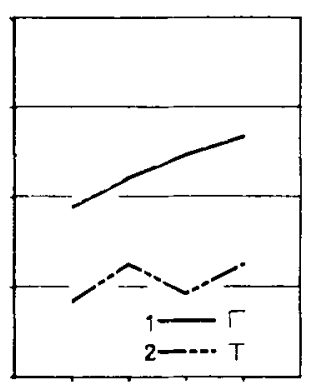

PAIR 1.5

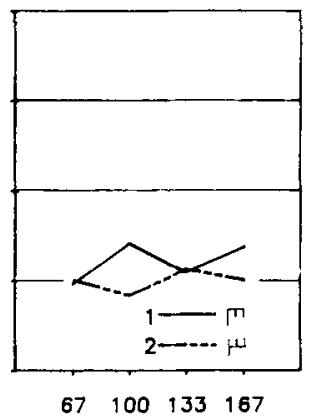

PAIR 1.3

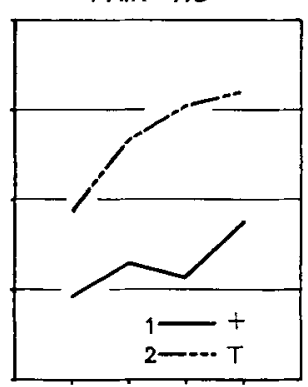

PAIR 7.6

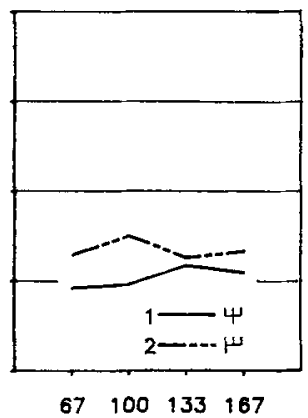

STIMULUS DURATION IN MSEC.

Figure 5. The discriminability of each micropattern pair used in Experiment 1 shown as a function of stimulus duration and which member of each pair played the role of the foreground.

differences between textures, whereas low accuracy at short stimulus durations followed by improved performance at longer stimulus durations indicates smaller perceptual differences. The present data are consistent with the basic idea that texture discrimination is not an all-ornone process. However, the asymmetry creates problems for the simple model, because the curves in Figure 5, Panel 1, for example, suggest that there are large-scale differences between $\mathrm{Ls}$ and $+\mathrm{s}$ but small-scale differences between $+s$ and Ls. Therefore, the model would have to be modified to account for the present data.

\section{EXPERIMENT 2}

In this experiment, we compared the discriminability of textures whose constituent micropatterns varied only in the spatial arrangement of line segments (see Figure 6, Pairs 2.1, 2.2, and 2.3) with that of textures whose constituent micropatterns had different numbers of line segments, terminators, and line crossings, yet retained the same overall shape in terms of their convex hulls (Pairs $2.4,2.5$, and 2.6). Because micropatterns with the same convex hulls will also be enclosed by the same circle, texton differences are not confounded with size differences. Note that Pairs 2.4 and 2.5 have intensity differences because of differences in the number of lines comprising the patterns of the pairs. Of course, texton theory says that the arrangement of line segments has at best a minimal effect on discrimination and consequently predicts that Pairs 2.1 to 2.3 should be more poorly discriminated than
Pairs 2.4 to 2.6. If factors associated with configurational differences are important, then the prediction is opposite to that of texton theory.

\section{Method}

Twelve more subjects were drawn from the subject pool described in Experiment 1; they were paid as before. Except for 1 subject, who reported having only monocular vision, the subjects reported normal or corrected-to-normal vision. With the exception of the micropatterns used, the materials, apparatus, and procedure were all identical to those described in Experiment 1.

\section{Results and Discussion}

The analysis of Experiment 2 was carried out in exactly the same manner as that described for Experiment 1.

Micropattern pairs. There was a significant effect of micropattern pairs $[F(6,66)=33.38, p<.0001]$; this is summarized in Figure 7. All micropattern pairs were significantly different from the control condition $[F(1,11)$

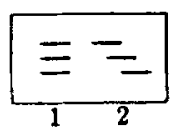

2.1
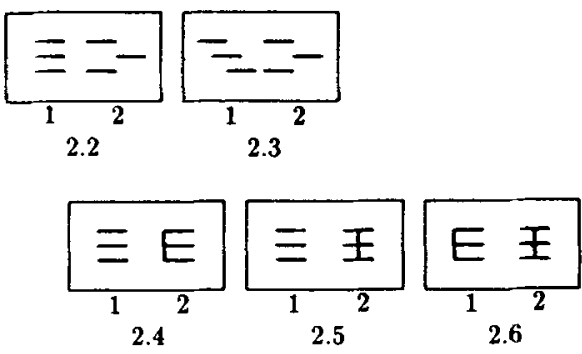

Figure 6. The six micropattern pairs used in Experiment 2. 


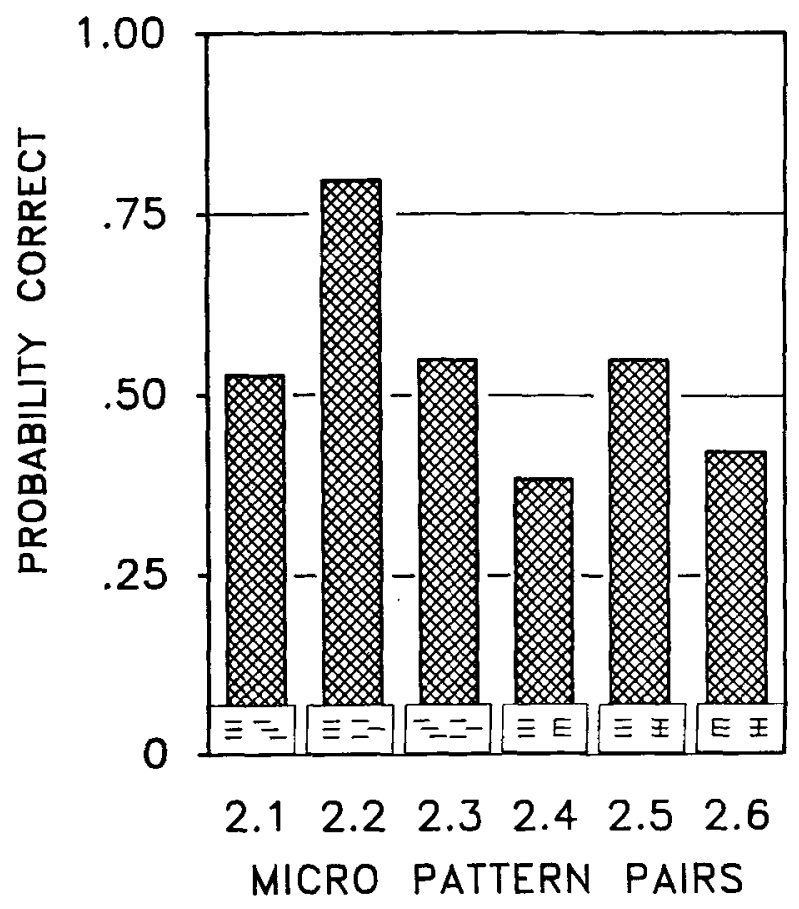

Figure 7. The overall discriminability of the micropattern pairs used in Experiment 2, collapsed over stimulus duration and the foreground/background factor.

$=43.19,85.85,21.8,16.27,26.64,29.3$ for Pairs 2.1 to 2.6 , respectively; all $p s<.005$ ]. Pairs $2.1,2.2$, and 2.3 were, on the whole, significantly more discriminable than Pairs 2.4, 2.5, and 2.6 $[F(1,11)=29.52, p<.005]$. The argument that differences in line arrangement are more important than texton differences would have been stronger if Pair 2.5 had elicited discrimination comparable to that of Pairs 2.4 and 2.6 rather than Pairs 2.1 and 2.3. However, it would be difficult to attribute the discriminability of Pair 2.5 to texton differences. Pairs 2.4 and 2.6 each have four unshared textons, whereas Pair 2.5 has only two. Therefore, the ease with which Pairs 2.4 and 2.6 are discriminated shows an almost perfect negative correlation with number of unshared textons. This is difficult to reconcile with the modified, textonsin-context version of texton theory.

Foreground/background effect. Figure 8 shows the breakdown of the micropattern pairs into their foreground/background components. There is a strong asymmetry in Pair $2.1[F(1,11)=41.89, p<.0002]$ and moderate effects for Pairs 2.2 and $2.5[F(1,11)=15.58$, 10.73 , respectively, both $p s<.01]$. For Pairs 2.1 and 2.2 , the asymmetry is related to micropattern size, with the larger patterns (as measured by the minimum enclosing circle) showing greater discriminability. For Pair 2.5, the pattern with more line segments $(2.5 .2)$ shows greater discriminability.

Stimulus-duration-trend analysis. The cell-by-cell breakdown of the experiment is shown in Figure 9. Sig- nificant linear trends were found for Conditions 2.1.2, 2.2.1, 2.2.2, 2.3.1, 2.3.2, 2.4.1, 2.5.2 $[F(1,11)=14.94$, $12.86,10.92,34.93,14.51,16.72,16.12$, respectively; all $p s<.01$ ], indicating significant improvement with increased processing time. Again, the range of performance should be noted.

Most writings about texton theory emphasize the role of the line segments, terminators, and line crossings that define the micropatterns. The way in which micropatterns differently stimulate large-scale blob detectors (in terms of the texton-theory notion of blobs) really is acknowledged only in passing. For example, Julesz (1984, p. 594) says, with no apparent empirical support, "Usually the effect of these dual textons (of large white blobs) on perception is small." The point here is that if some modified version of texton theory is to account for these data, then clearer definitions of textons are required and the conditions under which the various texton types will elicit discrimination must be specified.

\section{EXPERIMENT 3}

The micropatterns used in Experiment 3 are those shown in Figure 10. Pairs 3.1 and 3.2 have been used extensively by Julesz as examples of discriminable isodipole textures differing in number of terminators. Although the members of each pair are enclosed by the same circle, they have different configurational descriptions in terms of the shapes of their convex hulls. Julesz (1984) attaches only minor importance to these shape differences

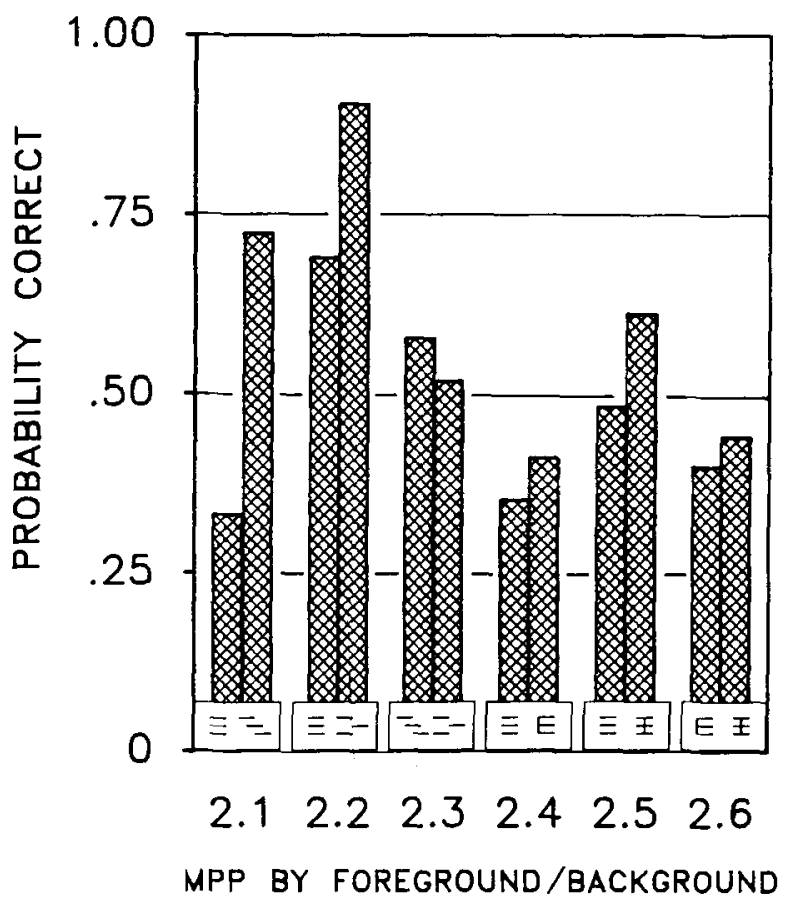

Figure 8. The discriminability of micropattern pairs used in Experiment 2 broken down in terms of which member of the pair played the role of the foreground. 

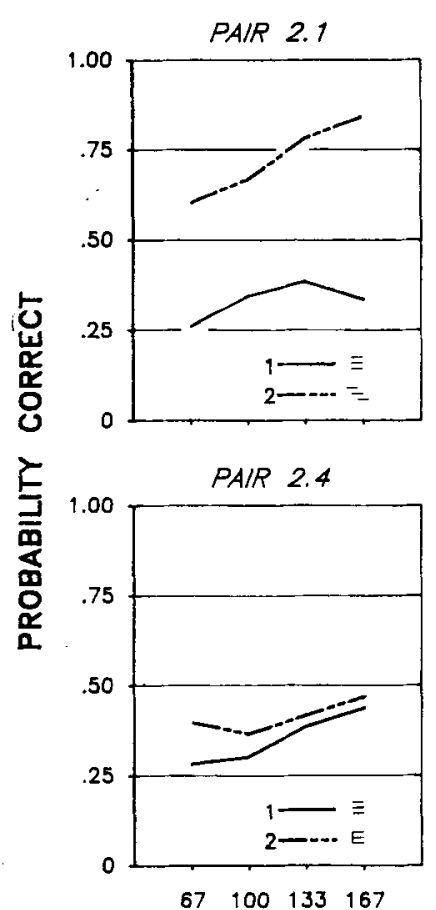

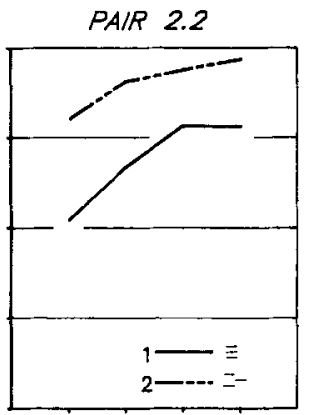

PAIR 2.5

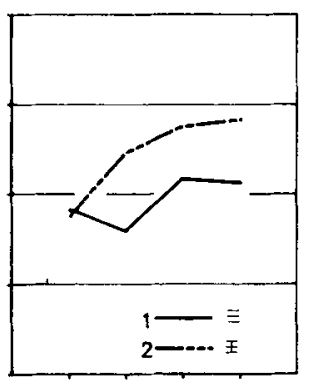

$67 \quad 100 \quad 133 \quad 167$

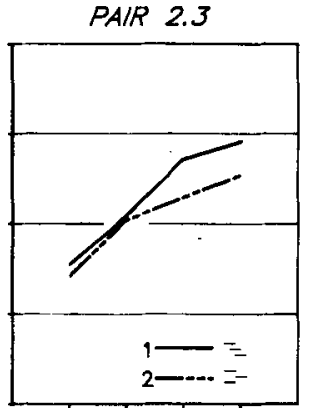

PAIR 2.6

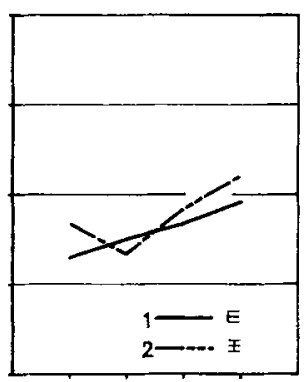

$\begin{array}{llll}67 & 100 & 133 & 167\end{array}$

STIMULUS DURATION IN MSEC.

Figure 9. The discriminability of each micropattern pair used in Experiment 2 shown as a function of stimulus duration and which member of each pair played the role of the foreground.

as determinants of discrimination when he says that weak learning effects may occur "provided that the convex hulls of the identical texton patterns to be discriminated have very different elongated blob shapes"' (p. 605). The patterns of Pair 3.3 differ in terms of overall size and shape but have identical line-segment and terminator makeup. Pairs $3.4,3.5$, and 3.6 have the same rectangular shape, but the members of each pair differ in terms of linesegment, line-crossing, and terminator makeup. If, as texton theory claims, shape differences defined by differences in the shape of the convex are of only minor importance, then, on the usual definitions of textons, only Pair 3.3 should be poorly discriminated.

\section{Method}

The 12 subjects in this experiment were drawn from the same pool described in Experiment 1. All subjects reported normal or
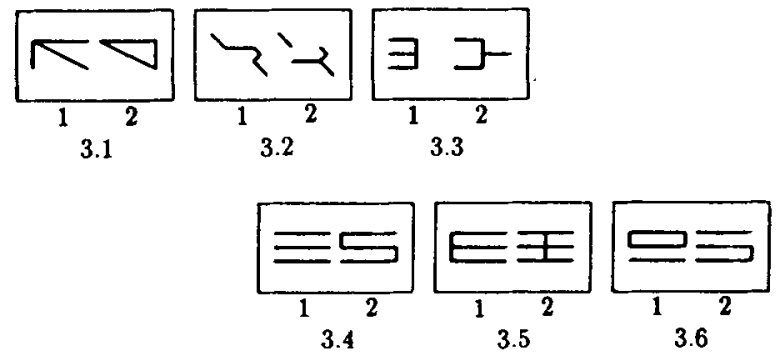

Figure 10. The six micropattern pairs used in Experiment 3. corrected-to-normal-vision. Except for the micropattern pairs used, the experimental method was exactly as described in Experiment 1.

\section{Results and Discussion}

The analysis of Experiment 3 was carried out in exactly the same manner as described in Experiment 1.

Micropattern pairs. The main effect of micropattern pairs, as summarized in Figure 11, was found to be significant $[F(6,66)=78.61, p<.0001]$ and Pairs 3.1, 3.2, and 3.3 were significantly more discriminable than Pairs 3.4, 3.5, and 3.6 $[F(1,11)=109.12, p<.0001]$. Planned (nonorthogonal) comparisons indicated that Pairs 3.1, 3.2, 3.3, and 3.5 were significantly different $[F(1,11)=15.06,6.51,144.24,12.31$ for Pairs 3.1, 3.2, 3.3 , and 3.5, respectively; all $p s<.01$ ] from the control group. An examination of Figure 11, however, reveals that only Pair 3.3, whose elements differ only in linesegment arrangement, was strongly discriminable (81\% discriminability averaged over all stimulus durations). Pairs 3.1 and 3.2 (supposedly classic examples of discriminable isodipole textures) showed poor overall discriminability $-40 \%$ and $34 \%$ discriminability, respectively, averaged over all stimulus durations. Performance was poorest for Pairs 3.4 and 3.6, which have many characteristics in common with the pair shown in Figure 1f. This suggests that the poor discriminability of the S- and 10-shaped micropatterns of Figure 1f is not due to similar line-segment and terminator composition but to general similarity of shape; that is, Pairs 3.4 and 


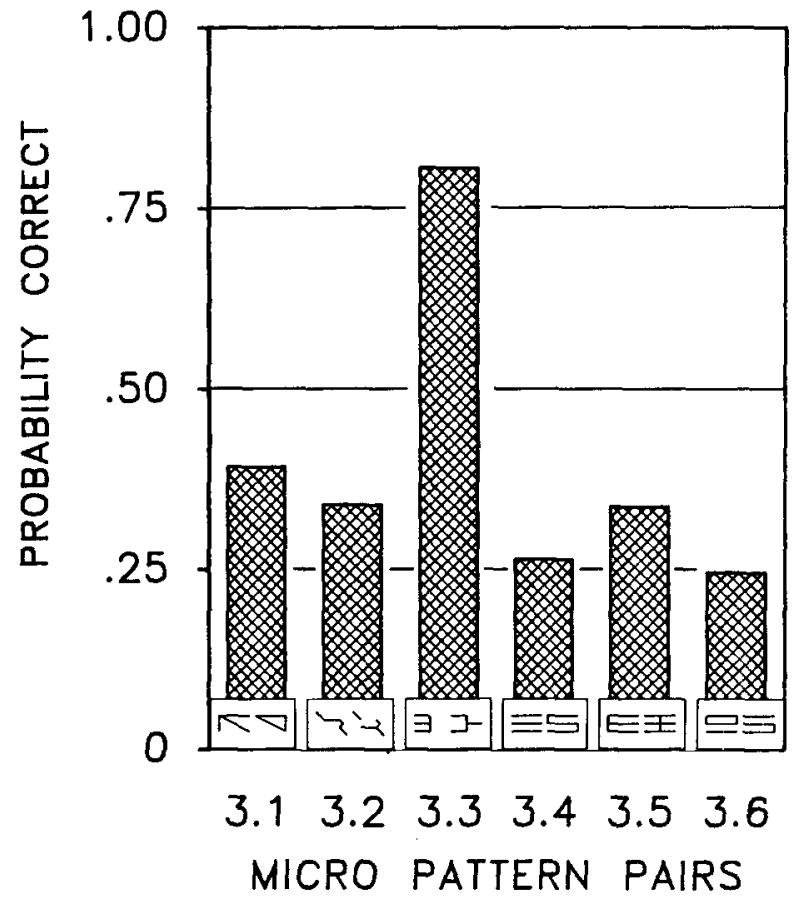

Figure 11. The overall discriminability of the micropattern pairs used in Experiment 3, collapsed over stimulus duration and the foreground/background factor.

3.6, which also have the same general rectangular shape yet differ in terminators and line segments, are also hard to discriminate. Pair 3.5 elicited the best performance of the three rectangle-shaped micropattern pairs.

Foreground/background effect. Although planned comparisons revealed that Pairs 3.1, 3.3, and 3.5 showed a significant foreground/background asymmetry $[F(1,11)$ $=8.18,15.89$, and 11.66 for Pairs 3.1, 3.3, and 3.5, respectively; all $p s<.02$ ], the effect was not quite as pronounced in numerical terms as it was in Experiment 1 (see Figure 12).

Stimulus-duration-trend analysis. Figure 13 shows the further breakdown of the experiment into its stimulus duration $\times$ micropattern $\times$ foreground/background components. Significant linear trends were found for Conditions 3.1.1, 3.2.1, 3.3.1, 3.3.2, and 3.5.2 $[F(1,11)=$ $30.42,6.51,30.76,28.71$, and 8.01 , respectively; all $p s<.05]$. Thus, performance improved in these cases with increased processing time. Of interest is the improvement in Pattern 3.1.1 at longer stimulus durations. This result, based on one version of the preattentive/attentive distinction, suggests a fast serial process rather than a distributed parallel process.

\section{EXPERIMENT 4}

We were concerned by our failure to replicate the classic discriminable isodipole results in Experiment 3. Subjects' difficulty with these patterns was surprising, since pilot work using a highly practiced subject had shown that these micropatterns were in fact easily discriminated. This suggests that a subject's experience with the micropatterns is a crucial aspect of performance in the texturediscrimination paradigm. In Experiment 4, we investigated this issue by tracking the performance of a relatively naive subject over 10 experimental sessions and then compared these results with those obtained from a very experienced subject.

\section{Method}

Subjects. One relatively inexperienced subject (P.G.) and 1 highly practiced subject (R.G.) were used in this experiment. P.G. had participated in Experiment 2 and had attained above average performance. P.G. was paid for participating in this study.

Materials. The micropattern pairs used were those shown in Figure 14. Note that Pairs 3.1, 3.2, 3.4, 3.5, and 3.6 had been used in Experiment 3. The isodipole pair, 4.3, replaced Pair 3.3 from Experiment 3.

Procedure. The method of presentation was essentially as before with two minor variations. First, the control condition was dropped from the experiment, so six pairs in total were used. Second, a no-mask condition was added. In this condition, the texture display stayed on for $167 \mathrm{msec}$ and was followed by $500 \mathrm{msec}$ of blank screen, during which the mask would ordinarily be seen, before the response box appeared. These changes to the design resulted in 480 trials. P.G. was tested on 5 consecutive nights. Each night he was run through two 480-trial sessions. R.G. was tested in five consecutive sessions in one morning.

\section{Results and Discussion}

We found that P.G.'s performance improved from approximately $40 \%$ correct over all conditions in Session 1

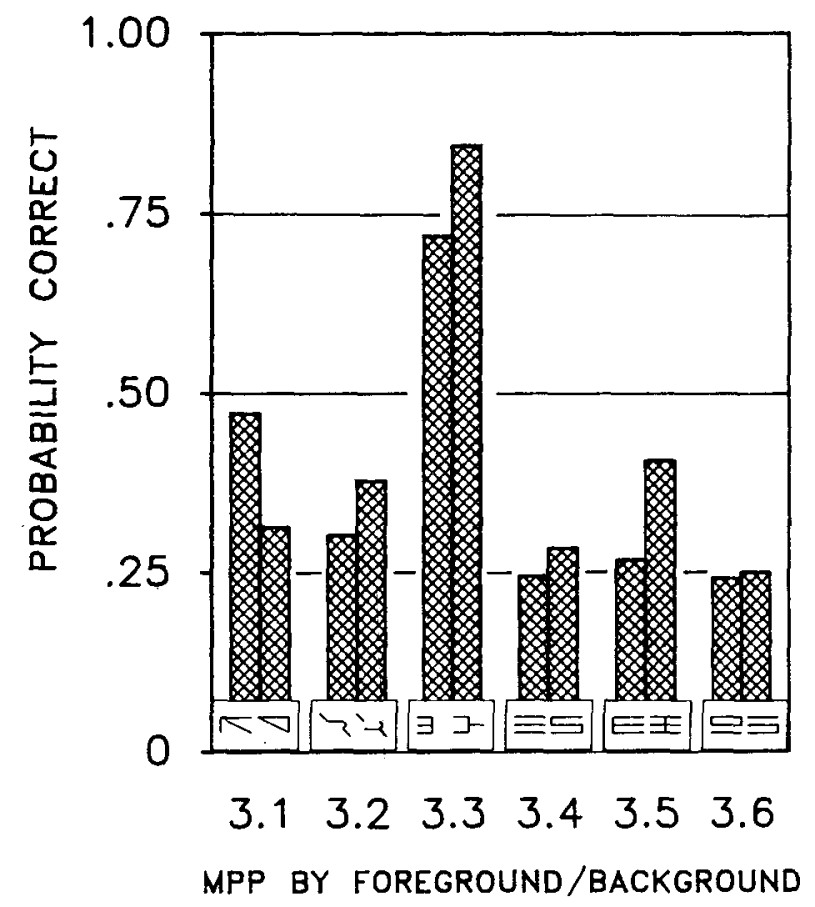

Figure 12. The discriminability of micropattern pairs used in $\mathrm{Ex}$ periment 3 broken down in terms of which meanber of the pair played the role of the foreground. 


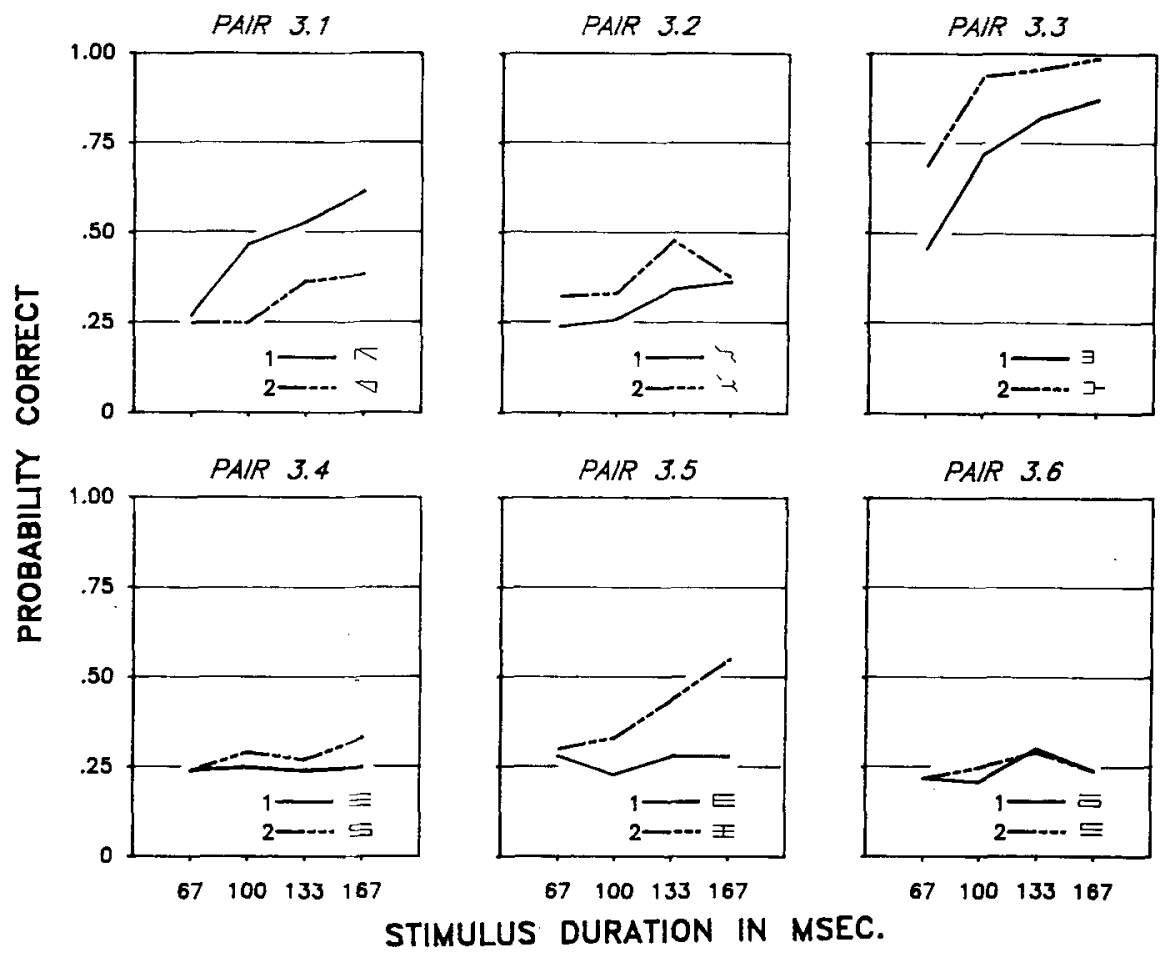

Figure 13. The discriminability of each micropattern pair used in Experiment 3 shown as a function of stimulus duration and which member of each pair played the role of the foreground.

to approximately $52 \%$ over all conditions in Session 2 . Thereafter, overall performance was slightly lower and only on Session 10 did overall performance reach a high of $55 \%$. Therefore, only the averaged results from the last five sessions are reported here.

An important point about practice can be made by simply considering the overall discriminability of micropattern pairs for the 2 subjects. Figure 15 shows the performance of the 2 subjects for each of the six micropattern pairs. The diagonally hatched bars represent R.G.'s data, and the horizontally and vertically hatched bars represent P.G.'s data.

With the exception of Pair 3.1, P.G.'s data do not differ markedly from the average subject's data in Experiment 3 . P.G. does show improvement on Pair 3.1 over the average subject from Experiment 3. For this subject at least, simple practice per se does not result in a general improvement in performance; only very specific improvement oc-

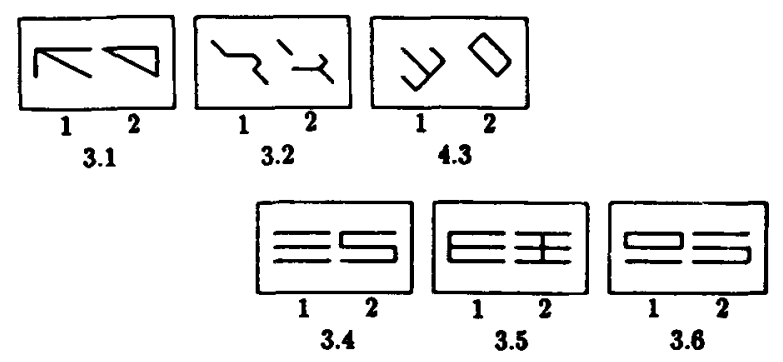

Figure 14. The six micropattern pairs used in Experiment 4. curred. R.G., however, does show a large advantage over P.G. and the average subject from Experiment 3 for all pairs except Pair 3.6. Pair 3.2, in particular, which P.G. and the average subject in Experiment 3 found very difficult, apparently poses no difficulty after massive practice and/or familiarity with the materials. Pairs 3.4 and 3.5 proved to be equally difficult for P.G. and the average subject from Experiment 3, whereas R.G. found Pairs 3.4 and 3.5 quite easy. On the whole, however, R.G. did find Pairs 3.1, 3.2, and 4.3 easier than Pairs 3.4, 3.5, and 3.6. We take this result to support our contention that the reported discriminability of Pairs 3.1 and 3.2 is not due to terminator differences. Given the very high performance of both P.G. and R.G. on Pair 3.1 and of R.G. on Pair 3.2, it is clear that sufficient information about the micropatterns is available to make highly accurate discriminations. However, great familiarity with these patterns is required before such discriminations can be made. We suggest that Julesz's claim that Pairs 3.1 and 3.2 are effortlessly discriminated is based on data obtained from highly practiced subjects and that the term "effortless" depends on the practice state of the subject. These pairs (3.1 and 3.2) differ from others that we investigated (e.g., $1.1,2.2 ., 3.3)$, which can be discriminated very accurately with relatively little practice.

\section{GENERAL DISCUSSION}

In the four experiments, we found performance in the texture discrimination task to be dependent on (1) the par- 


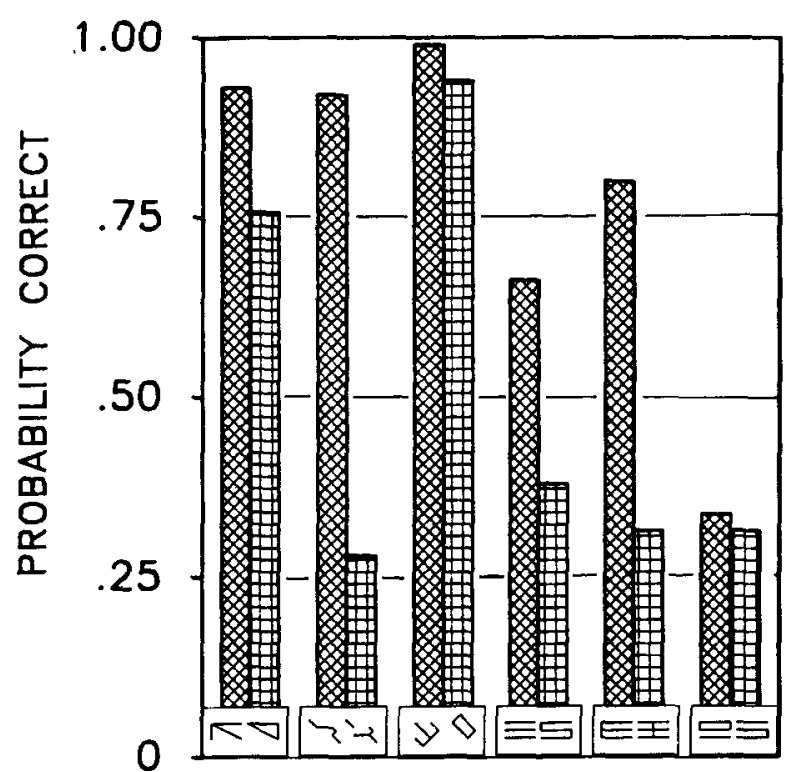

\section{$\begin{array}{llllll}3.1 & 3.2 & 4.3 & 3.4 & 3.5 & 3.6\end{array}$ MPP BY SUBJECTS}

Figure 15. The overall discrimimability of each micropattern pair wed in Experiment 4 collapsed over stimulus duration and the foreground/background factor. Subject R.G.'s results are shown as the diagonally hatched bars. P.G.'s results are shown as the vertically and horizontally hatched bars.

ticular micropattern pair used, (2) which member of the pair forms the foreground and which the background, (3) the time available to inspect the image, and (4) the familiarity that a subject has with the materials and procedure. In the following paragraphs, we will comment briefly on each of these issues.

We argued at the outset that the line-crossing and terminator textons had not been demonstrated to be independent of configurational differences between micropatterns. It was found that when configurational differences were minimized (in Experiment 1 by generating micropatterns from the same fundamental structure and in Experiments 2 and 3 by generating micropatterns with the same convex hulls), only one micropattern pair (2.5) with terminator, line-crossing, or line-segment differences was more discriminable overall than the L-T pair claimed to be "preattentively" indiscriminable by Julesz (1984). For this reason, we are skeptical about the proposition that line crossings and terminators play an important functional role in texture discrimination.

What, then, does permit discrimination? This question is incomplete as stated because its answer depends at least on the practice state of the subject, the question of which pattern is embedded in which, and the time available to inspect the image. However, with the results of naive subjects averaged over all stimulus durations and the foreground/background condition, we did find evidence that something related to the notion of micropattern size has an effect on overall discriminability.
At several points we have drawn attention to the correlation between discriminability and differences in micropattern size based on the minimum enclosing circle measure. However, we are not suggesting that the visual system computes the minimum enclosing circle for each micropattern in the display. Rather, we are suggesting that when two micropatterns that are composed of the same line segments are enclosed by different circles, they will stimulate different sets of simple receptors. Several recent demonstrations support the view that differences in rather simple, measurable image properties may be related to the discriminability of many textures. Gurnsey and Browse (1986), for example, pointed out that micropatterns composed of the same line elements (e.g., L and + ) have different gray level histograms when subjected to Gaussian blurring. In many cases, the magnitude of the differences between the histograms of these blurred textures predicted performance. Bergen (1986) showed that textures composed of Ls and $+s$ elicited different responses from circularly symmetric center-surround operators, and that, following an averaging procedure to spread activity throughout a local neighborhood, a simple thresholding operation could be applied to yield segmentation. Also, Beck (1986) showed that the responses of simple center-surround operators could form the basis for segmentation.

Such proposals have two (nonindependent) points to recommend them: (1) their simplicity is attractive, and it makes a great deal of sense to see just how much of textural discrimination can be accounted for in terms of simple operators, and (2) because we can be explicit about the computational form of these filters, they lend themselves to more rigorous evaluation than do suggestions about features for which no such definitions are forthcoming. For example, it is not clear how one would construct a line-crossing or terminator detector, and the term "elongated blob" seems too flexible to have any explanatory power. On the other hand, it is possible to implement and evaluate a scheme based on center-surround operators (e.g., Marr \& Hildreth, 1980; see also Fleet, Hallett, \& Jepson, 1985, for an analysis of a spatiotemporal centersurround operator) or orientation-selective filters (e.g., Fleet \& Jepson, 1985; Pollen \& Ronner, 1983). It should be noted that Beck has consistently argued that the primary determinants of textural segmentation are simple (measurable) physical variables such as scale, orientation, and brightness. We feel that progress will be made in understanding texture discrimination if informal definitions of features are replaced with explicit proposals about how measurable image properties relate to discriminability. Also, the computational mechanisms that utilize these measurements to produce a segmentation must be specified. Recent articles by Caelli (1985) and Grossberg and Mingolla (1985) have taken this more formal approach.

The usual view in texture research has been that textures may be conceptualized as points in an $n$-dimensional "feature space" and the hunt has been on for the features or image properties that define this space. It would be reasonable to expect, on this view, that the processing time 
required to discriminate two textures would be inversely related to the distance between the textures in the feature space. Consequently, performance should improve with exposure duration (Bergen \& Julesz, 1983), as was demonstrated in the present experiments. However, the usual view is also that textural segmentation involves computing differences between textural regions (e.g., Beck, 1982; Julesz, 1984). Recall that the third heuristic of texton theory states that attention is drawn to regions where texton differences (differences in local image properties) occur. If this were so, then there would be no reason to expect discrimination to be asymmetrical. A system that simply detects differences between textures should not exhibit asymmetries.

It may be, however, that the asymmetry is due to the fact that attention is drawn to regions with particular properties or to where the image changes in a particular way. The visual system must be sensitive to changes in the visual field, and these may elicit eye movements or covert attentional shifts (Posner, 1980). If certain image properties signal an attentional system, then it is reasonable to think of attention being drawn to the sudden appearance of elements that have a large magnitude on some dimension (e.g., scale ${ }^{5}$ or intensity). Now, if the subject's task is to detect the disparate quadrant, then having attention drawn to particular image regions could have different consequences, depending upon whether it was drawn to the foreground or the background. If attention is drawn to the disparate quadrant, then two possible consequences of this are (1) having attention drawn to a particular place can in itself form the basis for a decision about which is the disparate quadrant (i.e., since attention has been drawn here, this must be the disparate quadrant), or (2) the locus of processing (capable of detecting local feature differences) is shifted to the disparate region and perhaps stands a better chance of segmenting it from the background. If attention is drawn to some other quadrant, then either explanation (as to why being drawn to the correct quadrant would facilitate performance) implies that being drawn to the incorrect quadrant would have a deleterious effect on performance.

Although performance may be initially influenced by "attentional capture" (yielding the asymmetry), we would expect that with longer exposure durations performance would be determined by processes that detected differences between regions in the usual sense (yielding improved performance with increased processing time). Such a "two-process" account could explain the counterintuitive result that Ls embedded within Ts are more easily discriminated than Ts embedded within Ls (see Figure 4). In both cases, it would be assumed that some property of the L-shaped micropatterns captures attention. In this way, when the L-shaped micropatterns form the disparate region, they may stand a better chance of being correctly identified as such. When the L-shaped micropatterns form the background, they may be described as masking the $\mathrm{T}$ - or +-shaped micropatterns, as the case may be. With casual viewing, however, attentional capture may not $o c-$ cur and the differences between the $L$ - and +-shaped micropatterns would not be masked in the manner suggested.

Extensive experience with textures was shown to have a substantial effect on performance in the discrimination task. One question is whether highly practiced subjects (1) simply become more sensitive to the outputs of simple filters, or (2) develop strategies, or visual routines, that make a filter-based approach untenable. For example, we would not want to describe other rapid spatial processing behaviors, such as reading, in terms of simple filters and thresholds. In any case, it is clearly inadequate to talk about the discriminability of two textures without making reference to the practice state of the subjects involved. The challenge is for theories of texture discrimination, by being explicit enough to be expressed as programs that actually produce a segmentation of a textural display (Caelli, 1985), to account for the variety of factors that influence discrimination.

\section{REFERENCES}

BECK, J. (1966). Effect of orientation and shape similarity in perceptual grouping. Perception \& Psychophysics, 1, 300-302.

Beck, J. (1967). Perceptual grouping produced by line figures. Perception \& Psychophysics, 2, 491-495.

Beck, J. (1973). Similarity grouping of curves. Perceptual \& Motor Skills, 36, 1331-1341.

BECK, J. (1982). Textural segmentation. In J. Beck (Ed.), Organization and representation in perception. Hillsdale, NJ: Erlbaum.

BECK, J. (1986, June). Unresolved issues in texture segregation. Paper presented at the Center for Visual Science Symposium, Rochester, NY.

BECK, J., \& AMBLER, J. (1972). Discriminability of differences in line slope and in line arrangement as a function of mask delay. Perception \& Psychophysics, 12, 33-38.

BeCK, J., \& AMBler, J. (1973). The effects of concentrated and distributed attention on peripheral acuity. Perception \& Psychophysics, 14, 225-230.

Beck, J., Prazdny, K., Rosenfeld, A. (1983). A theory of textural segmentation. In J. Beck, B. Hope, \& A. Rosenfeld (Eds.), Human and machine vision. New York: Academic Press.

BERGEN, J. (1986, June). Texture segregation and the psychophysics of texture. Paper presented at the Center for Visual Science Symposium, Rochester, NY.

BERGEN, J., \& JuLESz, B. (1983). Rapid discrimination of visual patterns. IEEE Transactions on Systems, Man \& Cybernetics, SMC-13, 857-863.

Caelli, T. (1982). On discriminating visual textures and images. Perception \& Psychophysics, 31, 149-159.

CAELLI, T. (1985). Three processing characteristics of visual texture segmentation. Spatial Vision, 1, 19-30.

CAElli, T., \& Moraglia, G. (1985). On the discrimination of Gabor signals and discrimination of Gabor textures. Vision Research, 25, 671-684.

ENNs, J. (1986). Seeing textons in context. Perception \& Psychophysics, 39, 143-147.

Fleet, D. J., Hallet, P. E., \& JePson, A. D. (1985). Spatiotemporal inseparability in early visual processing. Biological Cybernetics, 52, 153-164.

FLEET, D. J., \& JEPSON, A. D. (1985). Measurement of visual information through hierarchical processing (Technical Report RBCV-TR85-8). Toronto: University of Toronto, Department of Computer Science.

Fox, J., \& MAYhEW, J. E. W. (1978). Texture discrimination and the analysis of proximity. Perception, 8, 75-91.

GrossberG, S., Mingolla, E. (1985). Neural dynamics of percep- 
tual grouping: Textures, boundaries, and emergent segmentations. Perception \& Psychophysics, 38, 141-171.

GuRNSEY, R., \& Browse, R. (1986, April). Comments on texton theory. Paper presented at the CIAR workshop on vision, London, Ontario.

HARALICK, R. M. (1979). Statistical and structural approaches to texture. Proceedings of the IEEE, 67, 786-804.

JOLICOEUR, P. (1986). Routine computations in the perception of spatial relations. Paper presented at the CIAR workshop on vision, London, Ontario.

Jolicoeur, P., Ullman, S., \& Mackay, M. (1986). Curve tracing: A possible basic operation in the perception of spatial relations. Memory \& Cognition, 14, 129-140.

JULESZ, B. (1980). Spatial nonlinearities in the visual perception of textures with identical power spectra. Philosophical Transactions of the Royal Society of London, B 290, 83-94.

JuLESZ, B. (1981). Textons, the elements of texture discrimination, and their interactions. Nature, 290, 91-97.

Julesz, B. (1984). Toward an axiomatic theory of preattentive vision. In G. Edelman, W. Einer, \& W. Cowan (Eds.), Dynamic aspects of neocortical function. New York: Wiley.

JuLESZ, B., \& BERGEN, J. (1983). Textons, the fundamental elements in preattentive vision and perception of textures. Bell Systems Technical Journal, 62, 1619-1645.

LAws, K. (1980). Textured image segmentation (Report 940). Los Angeles: USC Image Processing Institute.

MARR, D. (1982). Vision: A computational investigation into the human representation and processing of visual information. San Francisco: W. H. Freeman.

MARR, D., \& HiLdReTh, E. (1980). Theory of edge detection. Proceedings of the Royal Society, B 207, 187-217.

NeIsser, U. (1967). Cognitive psychology. New York: AppletonCentury-Crofts.

NothDURFT, H. C. (1985a). Discrimination of higher-order textures. Perception, 14, 539-543.

NothDURFT, H. C. (1985b). Orientation sensitivity and texture segmentation in patterns with different line orientation. Vision Research, $25,551-560$.

NothDURFT, H. C. (1985c). Sensitivity for structure gradient in texture discrimination tasks. Vision Research, 25, 1957-1968.

Olson, R. K., \& AtTneave, F. (1970). What variables produce similarity-grouping? American Journal of Psychology, 83, 1-21.

Pentland, A. P. (1984). Fractal-based descriptions of natural scenes. IEEE Transactions on Pattern Analysis \& Machine Intelligence, PAMI-6, 661-674.

Pollen, D. A., \& Ronner, S. F. (1983). Visual cortical neurons as localized spatial frequency filters. IEEE Transactions on Systems, Man \& Cybernetics, SMC-13, 907-916.

Pomerantz, J. R. (1981). Perceptual organization in information processing tasks. In M. Kubovy \& J. R. Pomerantz (Eds.), Perceptual organization. Hillsdale, NJ: Erlbaum.

Posner, M. I. (1980). Orienting of attention. Quarterly Journal of Experimental Psychology, 32, 3-25.

RILEY, M. (1981). The representation of image texture (Technical Report AI-TR-649). Cambridge, MA: Massachusetts Institute of Technology.

SCHATZ, B. (1977). The computation of immediate texture discrimination. Masters thesis, Department of Electrical Engineering and Computer Science, Massachusetts Institute of Technology.

Shulman, G. L., Remington, R. W., \& Mclean, J. P. (1979). Moving attention through visual space. Journal of Experimental Psychology: Human Performance \& Perception, 5, 522-526.
Treisman, A. M., \& Gelade, G. (1980). A feature integration theory of attention. Cognitive Psychology, 12, 97-136.

Treisman, A. M., \& Souther, J. (1985). Search asymmetry: A diagnostic for preattentive processing of separable features. Journal of Experimental Psychology: General, 114, 285-310.

Tsal, Y. (1983). Movements of attention across the visual field. Journal of Experimental Psychology: Human Performance \& Perception, 9, 523-530.

Ullman, S. (1984). Visual routines. Cognition, 18, 97-159.

\section{NOTES}

1. Many possible properties may be associated with the constructed, molar units: number of elementary features that the molar unit comprises, contrast, aspect ratio, and so forth. Whether or not these properties should be properly described as holistic is a matter we do not wish to debate. The point here is that Julesz rejects the notion that a constructive process follows the initial decomposition of the image and, of course, that the results of such a constructive process could have any influence on the discriminability of two textures. The view that configurational aspects of micropatterns are ignored in early vision appears to be inconsistent with Pomerantz's (1981) demonstrations of "failure of selective attention. " Nothdurft (1985a) has also produced evidence suggesting that the texture discrimination can occur in terms of "higher order" textural elements.

2. It is essential to note that texton detectors should be insensitive to the context in which their preferred patterns occur. A texton detector tuned to a vertical line segment should respond identically whether the line sengment was isolated in the visual field or part of an $\mathrm{L},+$, or $T$. If context affected the response of a texton detector or determined which detector was activated, then there would be no need to posit line crossings and terminators as textons, since the theory could account for discriminability on the basis of these differently stimulated line-segment texton detectors.

3. Throughout this paper, we consider the role of textons defined in the usual sense. Texton differences are considered differences in line segments, line crossings, and terminators, and not as differences in the overall blob-like characteristics of micropatterns (these we call configurational differences). What we are questioning, of course, is the assertion that configurational differences between micropatterns are of only minor importance in the texture-discrimination task. Whether configurational differences exert their putative influence through the stimulation of different simple filters or as a result of a constructive process (Beck, 1982; Marr, 1982) is not central to the argument. The issue is whether or not configurational differences play a role at all.

4. An alternative approach was used in Marr's lab by Riley (1981) and Schatz (1977; see also Nothdurft, 1985a, 1985b). The idea was to make performance dependent on some property of the disparate region, specifically, the form it defines. So, rather than simply detecting the existence of two regions, the subject had to say something about the shape of the regions; for example, was the shape a horizontal or a vertical rectangle? What letter did the disparate region define?

5. Consistent with this notion, Beck (1982) reported that it is easier to detect one long line among three short lines than to detect one short line among three long lines when viewed peripherally.

(Manuscript received July 17, 1986; revision accepted for publication November 20, 1986.) 